\title{
STONES IN DYSTOPIC KIDNEYS
}

Mohammed Elhadi MRCSEd MSc, Nisha Ranga, Shabi Ahmad FRCS Urol

${ }^{1}$ Sandwell and West Birmingham Hospitals. Birmingham, United Kingdom

Corresponding author Mohammed Ehadi: mohdessam1@hotmail.com

Submitted: March 9, 2017. Accepted: March 14, 2018. Published: April 27, 2018.

\begin{abstract}
Congenital abnormalities of the urinary tract comprise a variety of structural and functional malformations, these can range from mild asymptomatic to complex and life threatening. Renal anomalies predispose individuals to many complications, including recurrent urinary tract infections, stones formation, and impaired renal function. The knowledge of those anatomical variations is essential to urologists and trainees to help make correct diagnosis and offer appropriate treatment. Our aim is to define the role and evaluate the outcomes of flexible uretero-renoscopy (fURS) as an accepted treatment modality for urinary stone disease in 2 dystopic kidneys managed in our hospital.
\end{abstract}

Different morphological and rotational anomalies of the renal tract ${ }^{1}$ are well known risk factors for stone formation. These risk factors include: impaired drainage, urinary stasis, and an increased incidence of infections which can add real surgical challenges in stone management. Urologists and urology trainees are expected to provide understanding, demonstrate competencies and gain experience especially when dealing with such challenges. In this article we describe the surgical outcomes of ureterosopic stone treatment on patients with 2 rare conditions of laterally malrotated kidney and unilateral ectopic kidney treated endoscopically in our institute. Patients with these anomalies are often asymptomatic; those who symptomatic with pain, infection and haematuria require appropriate investigations and treatment. Here we illustrate findings of pre-, intra- and post-operative radiological images of those cases in order to draw clinicians' attention to these congenital abnormalities.

\section{CASE PRESENTATION}

The first patient is a 58-year-old male who referred via his general practitioner for investigations of persistence haematuria associated with left loin pain. Initial haematuria investigations did not reveal any sinister findings. Non-contrast computerized tomography was subsequently arranged, which has shown left lateral malrotated kidney with almost 10 millimetres stone lying in the renal pelvis (Figure 1). As result of the malrotation, lithotripsy was deemed inappropriate as fragments would have caused obstruction of ureter. Therefore, elective ureteroscopic and stone removal was arranged. The initial ureteroscopy was challenging owing to the tight ureter (Figure 2); as such a retrograde stent was inserted and a subsequent ureteroscopy was carried out. The stone subsequently fragmented and cleared (Figure 3 ). The second patient is a 29-year-old male who presented as an emergency with acute renal colic secondary to large obstructive stone in the right pelvi-ureteric junction in an ectopic pelvic kidney. The patient was initially under the general surgeons and had a computed tomography scan which did confirm the pathology (Figure 4). He underwent emergency retrograde double J stent insertion to relieve the obstruction. Subsequent elective ureteroscopic stone treatment was successfully carried out to clear the stone (Figure 5). 
FIG. 1 Non-contrast computed tomography showing the stone in the left malrotated kidney.

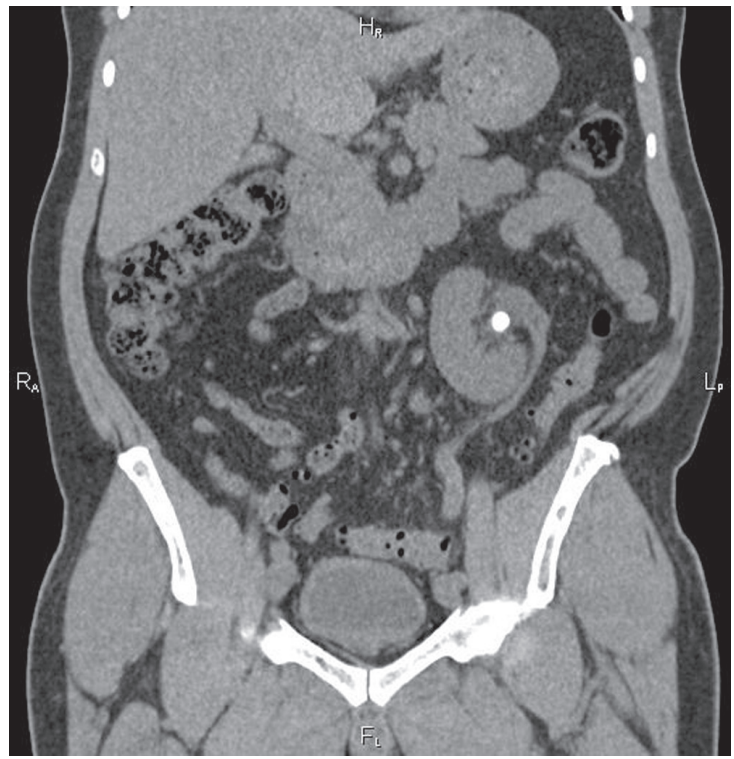

FIG. 3 Post-operative radiograph (kidney, ureter, bladder).

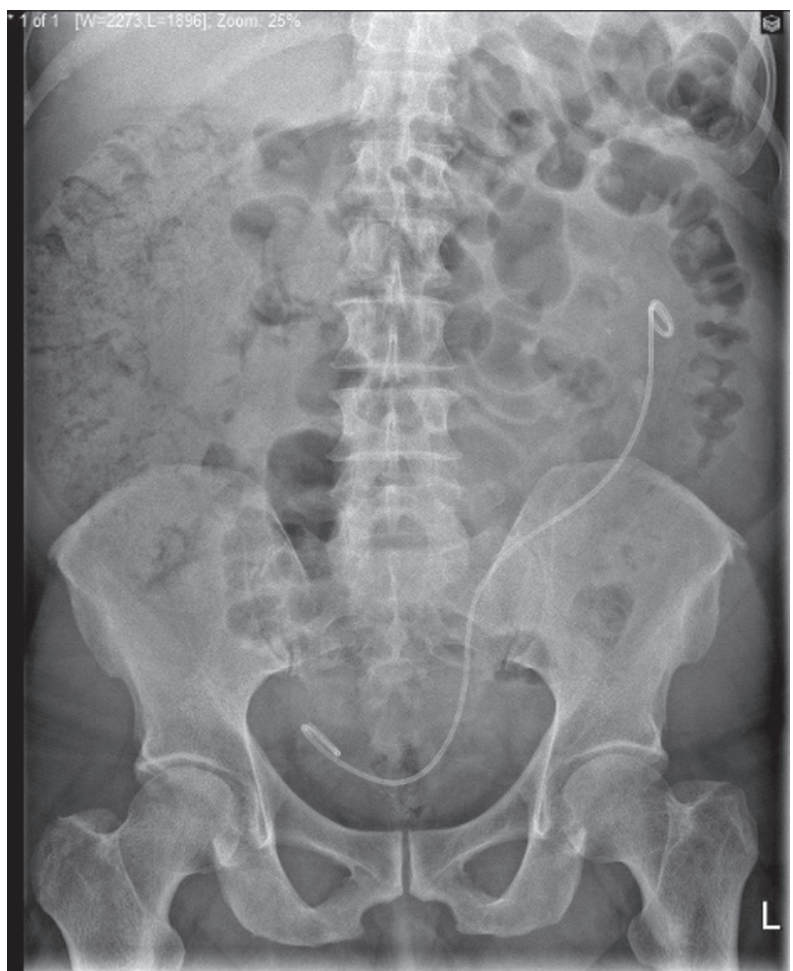

FIG. 2 Retrograde uretero-pyelogram demonstrate intraoperative findings of left lateral rotated kidney.

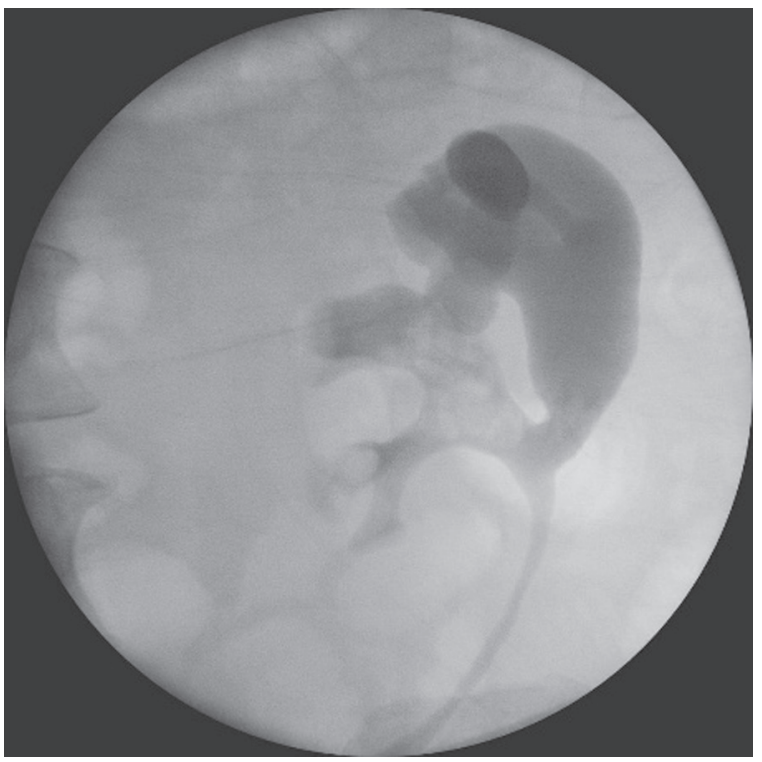

FIG. 4 Obstructing stone in the pelviureteric junction of right ectopic kidney.

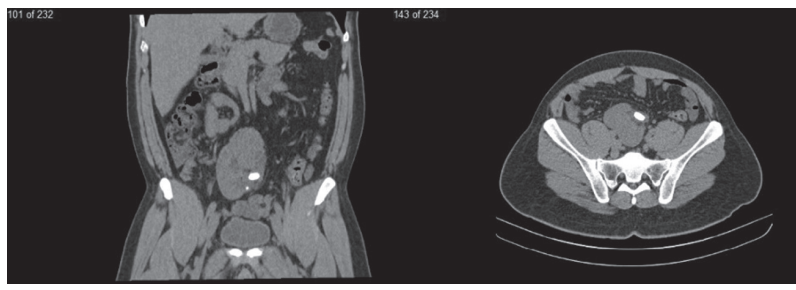

J Endolum Endourol Vol 1(1):e17-e20; April 27, 2018.

This article is distributed under the terms of the Creative Commons Attribution-Non Commercial 4.0 International License. (2018 Elhadi et al 
FIG. 5 Retrograde studies of right pelvic kidney.

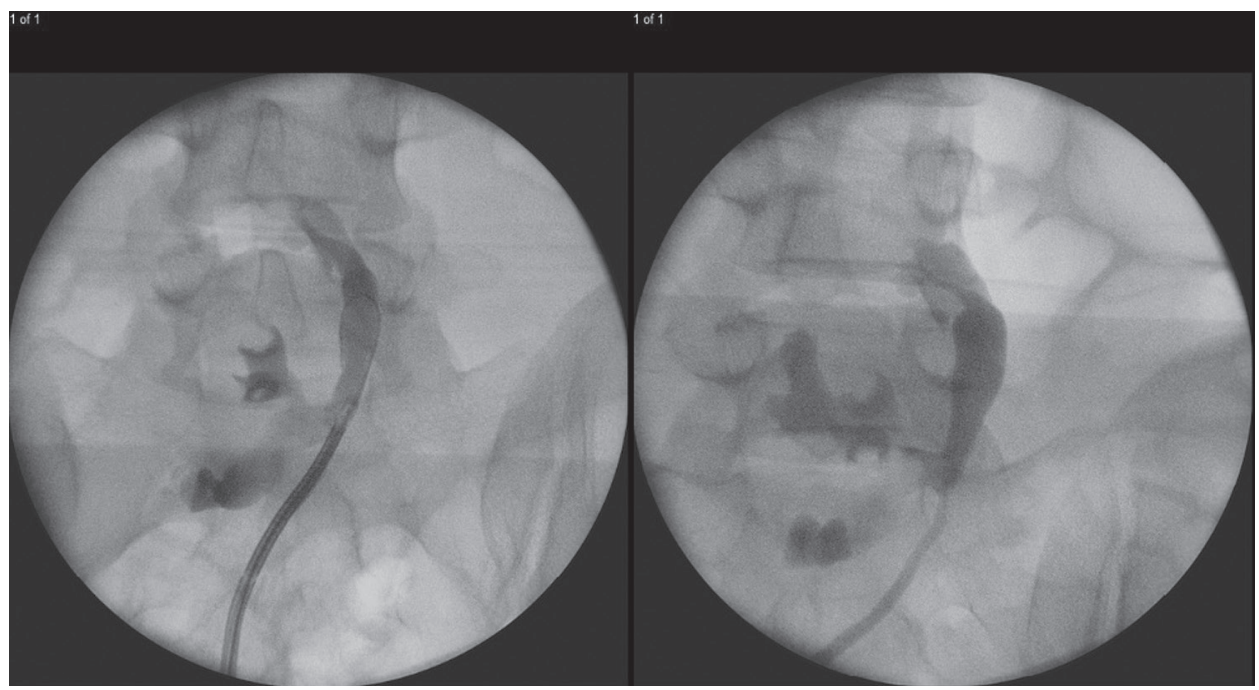

\section{DISCUSSION}

Renal ectopia and lateral malrotated kidney are very rare congenital abnormalities affecting the urinary tract with an incidence estimated at 1:12,000 and 1:2000 respectively. ${ }^{2,3}$ Due to impaired drainage, urinary stasis and recurrent infections, these congenital abnormalities predispose to stones formation through different metabolic defects such as hypercalcuria and hypocitraturia. ${ }^{4}$ Computerized axial tomography remained the key imaging modality to analyse and demonstrate detailed anatomical relationships of anomalous kidneys with the surrounding structures and organs. It also required prior to any planned treatment and to predict surgical outcomes. Shockwave lithotripsy, retrograde intrarenal surgery, percutaneous nephrolithotomy, open surgery and laparoscopy are different modalities which have been used to treat stones in congenital abnormal kidneys. ${ }^{5-7}$ However, international guidelines do not set a clear consensus on which treatment modality should be used to treat patients with those anomalies. ${ }^{8}$ Due to advanced technologies and varieties of surgical modalities to treat patients with urolithiasis, ureteroscopic stone removal is often a less preferred approach. This due to the challenges urologists may encounter in individuals with altered anatomy. Studies have shown that flexible ureterorenoscopy (fURS) is an effective modality for management of renal calculi less than $2 \mathrm{~cm}$ in kidneys with anomalies. ${ }^{9}$ The outcomes may vary depending on the type of the anomaly, stone burden, and stone location. ${ }^{10}$ From our experience we demonstrated the clinical challenges of diagnosing and treating stones in dystopic kidneys which have high educational values for both surgeons and trainees.

\section{REFERENCES}

1. Song R, Yosypiv IV. Genetics of congenital anomalies of the kidney and urinary tract. Pediatr Nephrol 2011 26:353-64.

2. Meizner I, Yitzhak M, Levi A, et al. Fetal pelvic kidney: a challenge in prenatal diagnosis? Ultrasound Obstet Gynecol 1995;5(6):391-3.

3. Pediatric uroradiology. Urologic radiology: A journal of diagnostic imaging. 1992;14(1):239-39.

4. Raj GV, Auge BK, Assimos D, Preminger GM. Metabolic abnormalities associated with renal calculi in patients with horseshoe kidneys. J Endourol 2004;18(2):157-61.

5. Salaheddin Y, Ionita D, Bengus F, et al. Percutaneous nephrolithotomy in patients with kidney malformations. Eur Urol Suppl [Internet]. 2017;16(11):e2968. Available from: http://linkinghub.elsevier.com/retrieve/pii/ S156990561732105X

J Endolum Endourol Vol 1(1):e17-e20; April 27, 2018.

This article is distributed under the terms of the Creative Commons Attribution-Non Commercial 4.0 International License. (C2018 Elhadi et al 
6. Matlaga BR, Kim SC, Watkins SL, Kuo RL, Munch LC, Lingeman JE. Percutaneous nephrolithotomy for ectopic kidneys: Over, around, or through. Urology 2006;67(3):513-7.

7. Sheir KZ, Madbouly K, Elsobky E, Abdelkhalek M. Extracorporeal shock wave lithotripsy in anomalous kidneys: 11-Year experience with two second-generation lithotripters. Urology 2003;62(1):10-5.

8. Ergin G, Kirac M, Unsal A, Kopru B, Yordam M, Biri H. Surgical management of urinary stones with abnormal kidney anatomy. Kaohsiung J Med Sci 2017;33(4):207-11.
9. Singh AG, Chhabra JS, Sabnis R, et al. Role of flexible uretero-renoscopy in management of renal calculi in anomalous kidneys: single-center experience. World J Urol 2017;35(2):319-24.

10. Legemate JD, Baseskioglu B, Dobruch J, et al. Ureteroscopic urinary stone treatment among patients with renal anomalies: patient characteristics and treatment outcomes. Urology 2017;110:56-62. doi: 10.1016/j. urology.2017.08.035. Epub 2017 Sep 4. 